\title{
Rapamycin protects the mitochondria against oxidative stress and apoptosis in a rat model of Parkinson's disease
}

\author{
JIANHUA JIANG $^{1^{*}}$, JUEAN JIANG ${ }^{2 *}$, YUANYI ZUO $^{1}$ and ZHENLUN GU ${ }^{1}$ \\ ${ }^{1}$ Department of Pharmacology, Medical College of Soochow University, Suzhou Institute \\ of Chinese Materia Medica, Suzhou 215007; ${ }^{2}$ Department of Special Requirements Ward, \\ The First Affiliated Hospital of Soochow University, Suzhou 215006, P.R. China
}

Received December 6, 2012; Accepted January 9, 2013

DOI: $10.3892 / \mathrm{ijmm} .2013 .1280$

\begin{abstract}
Parkinson's disease (PD) is a neurodegenerative disease, in which oxidative stress and mitochondrial dysfunction are responsible for neuronal apoptosis. Rapamycin plays a crucial role in reducing oxidative stress and protecting the mitochondria. However, its protective role in PD has not yet been fully elucidated. In this study, we report that pre-treatment with rapamycin provides behavioral improvements, protects against the loss of dopaminergic neurons, and alleviates mitochondrial ultrastructural injuries in a rat model of PD. Peroxide levels were lower and antioxidant activities were higher in PD rats pre-treated with rapamycin compared to the PD rats pre-treated with the vehicle. Furthermore, pre-treatment with rapamycin significantly elevated the expression of anti-apoptotic markers and reduced the levels of pro-apoptotic markers compared to pre-treatment with the vehicle. In conclusion, our results demonstrated that rapamycin reduced oxidative stress and alleviated mitochondrial injuries in the 6-hydroxydopamine (6-OHDA)-induced rat model of PD, which may subsequently contribute to its anti-apoptotic effects. The ability of rapamycin to exhibit neuroprotection in a rat model of PD may be related to its antioxidant capabilities.
\end{abstract}

Correspondence to: Professor Zhenlun Gu, Department of Pharmacology, Medical College of Soochow University, Suzhou Institute of Chinese Materia Medica, 708 Renmin Road, Suzhou 215007, P.R. China

E-mail: zhenlungu.2003@163.com

*Contributed equally

Abbreviations: PD, Parkinson's disease; ROS, reactive oxygen species; mTOR, mammalian target of rapamycin; 6-OHDA, 6-hydroxydopamine; L-R, low dose of rapamycin; M-R, moderate dose of rapamycin; $\mathrm{H}-\mathrm{R}$, high dose of rapamycin; $\mathrm{TH}$, tyrosine hydroxylase; TEM, transmission electron microscope; MDA, malondialdehyde; SOD, superoxide dismutase; GSH-PX, glutathione peroxidase; RT-PCR, reverse transcription-polymerase chain reaction

Key words: Parkinson's disease, rapamycin, oxidative stress, mitochondrial dysfunction, apoptosis

\section{Introduction}

Parkinson's disease (PD) is a common age-related, progressive neurodegenerative disease, characterized by the loss of dopaminergic neurons in the substantia nigra pars compacta $(\mathrm{SNpc})(1,2)$. Current treatments principally ameliorate clinical manifestations of the disease rather than prevent neuronal death, which reflects an incomplete understanding of the basis of PD (3). However, oxidative stress and mitochondrial dysfunction have been considered to be responsible for the pathological features of PD, such as neuronal death and apoptosis $(4,5)$. Reactive oxygen species (ROS) produced generated by oxidative stress are widely believed to contribute to the loss of dopaminergic neurons, mainly in the form of apoptosis (6). The mitochondria, the most important sites of ROS production, are crucial in the process of apoptotic regulation (7). The impairment of mitochondrial activity increases ROS levels and causes oxidative damage to proteins, lipids and DNA (8). Thus, the reduction of oxidative stress may alleviate mitochondrial damage and appears to be a promising strategy in the prevention and treatment of PD.

Rapamycin, a lipophilic, macrolide antibiotic, is widely used in clinical practice as an immunosuppressant. It induces autophagy by inactivating the mammalian target of rapamycin (mTOR) (9). Rapamycin binds intracellularly to the FK506 binding protein and targets mTOR to block the calcium-dependent and non-dependent mTOR signaling pathway (10). A number of studies have previously claimed that rapamycin is able to provide neuronal protection in a series of experimental models of neurodegenerative diseases. Ravikumar et al $(11,12)$ showed that rapamycin protects against mutant Huntington-induced degeneration in cell, fly and mouse models of Huntington's disease. Pre-treatment with rapamycin has been shown to prevent apoptosis and reduce ubiquitinated protein aggregation in differentiated PC12 cells. C57/BL mice post-treated with rapamycin significantly demonstrated an attenuated loss of dopaminergic neurons (13). A recent study illustrated that rapamycin but not FK506 protects neurons from death in both cellular and animal models of PD by blocking the translation of RTP801 (14). The mitochondrial protective effects of rapamycin have previously been introduced in Drosophila and this protection may be accounted for by the 
reduced mitochondrial load and enhanced mitochondrial clearance by autophagy (15). Certain studies have shown that rapamycin reduces oxidative stress in frataxin-deficient yeast cells (16) and restores the mitophagy inhibited by monoamine oxidase B (Mao-B) in an inducible cell model (17). However, other studies have indicated that rapamycin increases oxidative stress response in adult stem cells (18) and have shown that nucleolar disruption leads to oxidative damage and Parkinsonism through mTOR repression (19). These studies suggest that the protective role of rapamycin against oxidative stress in PD appears not to be fully clarified, and the mitochondrial injuries and apoptosis induced by oxidative stress remain to be elucidated.

In this study, we employed rapamycin in a rat model of PD induced by 6-hydroxydopamine (6-OHDA). Rotational behaviors, dopaminergic neuronal loss and mitochondrial injuries were analyzed. By detecting markers of oxidative stress and apoptosis in rapamycin-pre-treated PD rats, we demonstrate that rapamycin exerts protective effects on the mitochondria, preventing oxidative stress and apoptotic responses in a rat model of PD.

\section{Materials and methods}

Animals and induction of $P D$. Female Sprague-Dawley rats weighing 200-250 g were obtained from the Experimental Animal Center of Soochow University (Suzhou, China). All rats were housed in a temperature-controlled environment at $22 \pm 1^{\circ} \mathrm{C}$, with $12 / 12 \mathrm{~h} \mathrm{light/dark}$ cycle and allowed ad libitum access to food and water. All experimental procedures were approved by the Animal Care and Use Committee of Soochow University. For the induction of PD, the rats were intraperitoneally (i.p.) anesthetized with chloral hydrate $(400 \mathrm{mg} / \mathrm{kg})$. For stereotactic surgery, 6-OHDA (16 $\mu \mathrm{g}$ dissolved in $4 \mu \mathrm{l}$ $0.9 \%$ sodium chloride solution containing $0.2 \%$ ascorbic acid; Sigma) was injected into 2 different sites within the right striatum. Striatum injection coordinates were as follows: site 1: anterior-posterior (AP) $+0.7 \mathrm{~mm}$, medial-lateral (ML) $-3.0 \mathrm{~mm}$, dorso-ventral (DV) $-4.5 \mathrm{~mm}$; site 2: AP - $0.2 \mathrm{~mm}$, ML - $2.6 \mathrm{~mm}$, DV $-6.0 \mathrm{~mm}$. The sham-operated rats underwent the same surgical procedure in the absence of 6-OHDA. After the surgery, animals were kept in a temperature-controlled room for complete recovery.

Grouping, drug treatment and behavioral testing. The rats were randomly divided into the following 5 groups and each group consisted of 24 animals: i) Sham-operated rats treated as described above (group S); ii) PD model rats pre-treated with the vehicle in the absence of rapamycin (group P); iii) PD model rats pre-treated with a low dose of rapamycin (group L-R) $(0.05 \mathrm{mg} / \mathrm{kg} /$ day); iv) PD model rats pre-treated with a moderate dose of rapamycin (group M-R) $(0.5 \mathrm{mg} /$ $\mathrm{kg} / \mathrm{day}$ ); v) PD model rats pre-treated with a high dose of rapamycin (group H-R) $(5 \mathrm{mg} / \mathrm{kg} /$ day). Rapamycin (Bomeibio, Hebei, China) was dissolved in dimethyl sulfoxide (DMSO) and then diluted with $0.9 \%$ sodium chloride solution with the final concentration of DMSO at $0.5 \%(\mathrm{v} / \mathrm{v})$. Different doses of rapamycin or vehicle $(0.9 \%$ sodium chloride solution containing $0.5 \%$ DMSO) were intragastrically administered once daily to the rats from day 7 before the induction of PD.
Three weeks later, all animals underwent rotational testing (day 25) to evaluate the motor asymmetry caused by the unilateral nigrostriatal lesion. Each rat received R-(-)-Apomorphine hydrochloride hemihydrate (APO; Sigma) $(0.5 \mathrm{mg} / \mathrm{kg}$ i.p., dissolved in $0.9 \%$ sodium chloride solution) and was placed in a transparent cage immediately. Contralateral rotations $\left(360^{\circ}\right.$, in short axis) $30 \mathrm{~min}$ from the initiation of rotation were recorded.

Tyrosine hydroxylase (TH) immunohistochemistry. On day 28 , the rats were sacrificed by cardiac perfusion with a solution of $4 \%$ paraformaldehyde (PFA). The brains were removed, post-fixed in $10 \%$ formalin-PBS solution for 2 days, and processed for paraffin embedding. To evaluate $\mathrm{TH}^{+}$ neurons in the substantia nigra, we examined serial sections located in areas between -4.8 and $-5.8 \mathrm{~mm}$ from the bregma in the rostrocaudal direction, according to the Rat Brain Atlas by Paxinos and Watson (20). The brain sections were cut into $4 \mu \mathrm{m}$ slices. Following deparaffinization and rehydration, antigen retrieval was performed and endogenous peroxidase activity was blocked. After the sections were blocked with $3 \%$ normal goat serum, rabbit polyclonal antibody against TH (1:1,000 dilution; Sigma) was added for $1 \mathrm{~h}$ of incubation at $37^{\circ} \mathrm{C}$. The slices were washed prior to incubation with a horseradish peroxidase-conjugated secondary antibody (1:200 dilution; Dako, Carpinteria, CA, USA) at $37^{\circ} \mathrm{C}$ for $30 \mathrm{~min}$. The antibody-peroxidase complex was revealed by incubating the slices with a 3,3-diaminobenzidine peroxidase substrate kit (Sigma). A total of 8 sections per rat were analyzed for $\mathrm{TH}^{+}$neurons using an Olympus X71 photomicroscope. The measurements were carried out by 2 observers who were blinded to the source of the images. The number of ipsilateral dopaminergic neurons was presented as a percentage of $\mathrm{TH}^{+}$cells in the ipsilateral hemisphere vs. the contralateral one.

Transmission electron microscope (TEM) analysis. Following perfusion, the striatum were dissected and fixed in $2.5 \%$ glutaraldehyde in $0.01 \mathrm{M}$ PBS (PH 7.4) for $3 \mathrm{~h}$ at $4^{\circ} \mathrm{C}$, followed by post-fixation in $1 \% \mathrm{OsO}_{4}$ for $2 \mathrm{~h}$ at $4^{\circ} \mathrm{C}$, and then washed with the same buffer. Before being embeded in epoxy resin, the specimens were dehydrated in gradient series of ethanol (20-100\%) and acetone. Ultra-thin slices were cut using an ultramicrotome, collected on copper grids, and stained with uranyl acetate and lead citrate. The slices were observed using an Hitachi H-600 TEM.

Assays of peroxide levels and antioxidant activities. In order to determine peroxide levels of malondialdehyde (MDA) and the activities of antioxidant enzymes, including superoxide dismutase (SOD) and glutathione peroxidase (GSH-PX), the midbrain tissues were quickly removed and homogenized with $0.9 \%$ sodium chloride solution on day 28 . After being centrifuged, the supernatant were collected for bioassays using relative commercial assay kits (Jiancheng Bioengineering Institute, Nanjing, China) according to the manufacturer's instructions. MDA levels in the brain tissue were expressed as nanomole per milligram of protein $(\mathrm{nmol} /$ mg protein). The activity of SOD and GSH-PX was presented as units per milligram of protein (U/mg protein). 
Table I. Specific primers and their sequences used for RT-PCR analysis in this study.

\begin{tabular}{lll}
\hline Primer & \multicolumn{2}{c}{ Sequence } \\
\hline Bcl-2 & F: & 5'-CCGGGAGATCGATGAAGTA-3' \\
& R: & 5'-CATATTTGTTTGGGGCATGTCT-3' \\
Bax & F: & 5'-GCAGGGAGGATGGCTGGGGAGA-3' \\
& R: & 5'-TCCAGACAAGCAGCCGCTCACG-3' \\
GAPDH & F: 5'-GTCGTGGAGTCTACTGGCGTCTT-3' \\
& R: 5'-CAGTCTTCTGAGTGGCAGTGATGG-3'
\end{tabular}

RT-PCR, reverse transcription-polymerase chain reaction; F, forward; $\mathrm{R}$, reverse.

Reverse transcriptional-polymerase chain reaction $(R T-P C R)$. The striatum tissues were immediately removed and frozen in liquid nitrogen. Total RNA was extracted using TRIzol reagent (Invitrogen, Carslbad, CA, USA) according to our standardized laboratory protocol. The cDNA was synthesized with the PrimeScript ${ }^{\text {TM }}$ RT Master Mix (Takara Bio, Inc., Shiga, Japan) according to the manufacturer's instructions. PCR amplification was performed to analyze the gene expression. The specific primers used for PCR analyses are presented in Table I. Parallel amplification of rat GAPDH was performed as the endogenous control, and the intensity of each band was quantified using gel analysis software (SigmaScan Pro Image version 5.0). The gene expressions were presented as the ratio between the band intensity value for Bcl-2 or Bax and the value for GAPDH from the same RNA sample.

Western blot analysis. The rapidly removed striatum tissues were homogenized in ice-cold lysis buffer (containing 1\% phenylmethanesulfonyl fluoride). Each sample (containing $50 \mu \mathrm{g}$ protein) was mixed with the same volume of sample buffer and boiled for $5 \mathrm{~min}$. The proteins with different molecular weights were separated on 12-15\% SDS-PAGE gels and then transferred onto nitrocellulose membranes. The membranes were blocked with $5 \%$ non-fat dry milk in $0.1 \%$ TBST for $1 \mathrm{~h}$ at room temperature. Subsequently, the membranes were incubated with the following antibodies at $4{ }^{\circ} \mathrm{C}$ overnight: mouse anti-rat Bax and Bcl-2 (1:200 dilution; Santa Cruz Biotechnology, Inc., Santa Cruz, CA, USA), rabbit anti-rat cleaved caspase-9 (1:500 dilution; Cell Signaling Technology, Inc., Danvers, MA, USA), mouse anti-rat cytochrome $c(1: 1,000$ dilution; Santa Cruz Biotechnology, Inc.), and mouse anti-rat $\beta$-actin (1:1,000 dilution; Beyotime Biotechnology, Haimen, China). Goat anti-rabbit or goat anti-mouse fluorescenceconjugated secondary antibodies (1:20,000 dilution; Beijing Boisynthesis Biotechnology Co., Ltd., Beijing, China) were then applied for $1 \mathrm{~h}$ of incubation at room temperature and the immunofluorescence were detected with an Odyssey Nearinfrared two-color laser imaging system (Li-Cor; Lincoln, $\mathrm{NE}$ ). The optical density of each protein band was quantified by BandScan software version 5.0. The optical density of each protein band was normalized to the corresponding density of the $\beta$-actin band.
Statistical analysis. All statistical analyses were performed using SPSS 13.0 for Windows (SPSS Inc., Chicago, IL, USA). All quantitative data are presented as the means \pm standard deviation (SD). A Student's t-test or non-parametric MannWhitney U test was used to analyze the differences between independent samples. One-way ANOVA was initially performed to determine whether an overall statistically significant difference existed before using the Student's t-test. A value of $\mathrm{p}<0.05$ was considered to indicate a statistically significant difference.

\section{Results}

Rapamycin provides behavioral improvements in a rat model of $P D$. The injection of apomorphine did not produce rotational behaviors in the sham-operated group (group $S, n=24$ ), whereas the PD model group (group $\mathrm{P}, \mathrm{n}=24$ ) displayed a significant number of contralateral rotations $(406.67 \pm 67.72 / 30 \mathrm{~min})$. Pre-treatment with various doses of rapamycin significantly reduced the number of contralateral rotations in group L-R $(336.63 \pm 57.97 / 30 \mathrm{~min}, \mathrm{n}=24), \mathrm{M}-\mathrm{R}(348.75 \pm 55.82 / 30 \mathrm{~min}$, $\mathrm{n}=24)$ and $\mathrm{H}-\mathrm{R}(317.13 \pm 52.61 / 30 \mathrm{~min}, \mathrm{n}=24)$, as compared with those in group $\mathrm{P}(\mathrm{p}<0.05$ in all cases). However, there were no statistical differences in rotations observed between groups L-R, M-R and H-R ( $\mathrm{p}>0.05$ in all cases).

Rapamycin protects against the loss of dopaminergic neurons and mitochondrial ultrastructual injuries in a rat model of $P D$. The loss of dopaminergic neurons in the SNpc was examined by $\mathrm{TH}$ immunohistochemistry (Fig. 1A). The number of remaining $\mathrm{TH}^{+}$neurons in the $\mathrm{SNpc}$ from the $\mathrm{PD}$ model rats (group P) was significantly lower as compared with that in the $\mathrm{SNpc}$ from the control rats (group S) $(27.56 \pm 5.55$ vs. $92.37 \pm 5.56 \%, \mathrm{p}<0.001)$. Pre-treatment with a low (L-R, $47.19 \pm 7.94 \%, \mathrm{p}<0.001)$, moderate $(\mathrm{M}-\mathrm{R}, 43.17 \pm 7.09 \%$, $\mathrm{p}<0.001)$ or high $(\mathrm{H}-\mathrm{R}, 51.69 \pm 10.54 \%, \mathrm{p}<0.001)$ dose of rapamycin significantly increased the number of $\mathrm{TH}^{+}$neurons in the SNpc as compared to pre-treatment with the vehicle (group P) (Fig. 1B). Although the number of $\mathrm{TH}^{+}$neurons in group $\mathrm{H}-\mathrm{R}$ was higher than that in groups $\mathrm{L}-\mathrm{R}$ and $\mathrm{H}-\mathrm{R}$, there was no significant difference between them ( $p>0.05$ in all cases) (Fig. 1B). To analyze mitochondrial ultrastructural injuries, TEM analysis was performed. In the striatum of the control rats (group S), healthy neurons displayed normal nuclei with dispersed chromatin and mitochondria with double membranes and clear cristae. In the PD model rats (group P), the shrunken nuclei in the neurons demonstrated condensed chromatin (Fig. 2A), and the mitochondria were evidently swelled and vacuolated and the cristae were lessened, distorted or had even disappeared (Fig. 2B). However, in the rats pretreated with rapamycin (groups L-R, M-R and H-R), the nuclei in the neurons had less condensed chromatin and less swelled mitochondria with distinct cristae and decreased vacuolations, as compared with those in the rats pre-treated with the vehicle (group P) (Fig. 2). Ultrastructural manifestations of neurons appeared to be comparable between the groups pre-treated with various doses of rapamycin.

Rapamycin reduces oxidative stress in a rat model of $P D$. In the rats in group P, MDA levels were elevated as compared with 
A
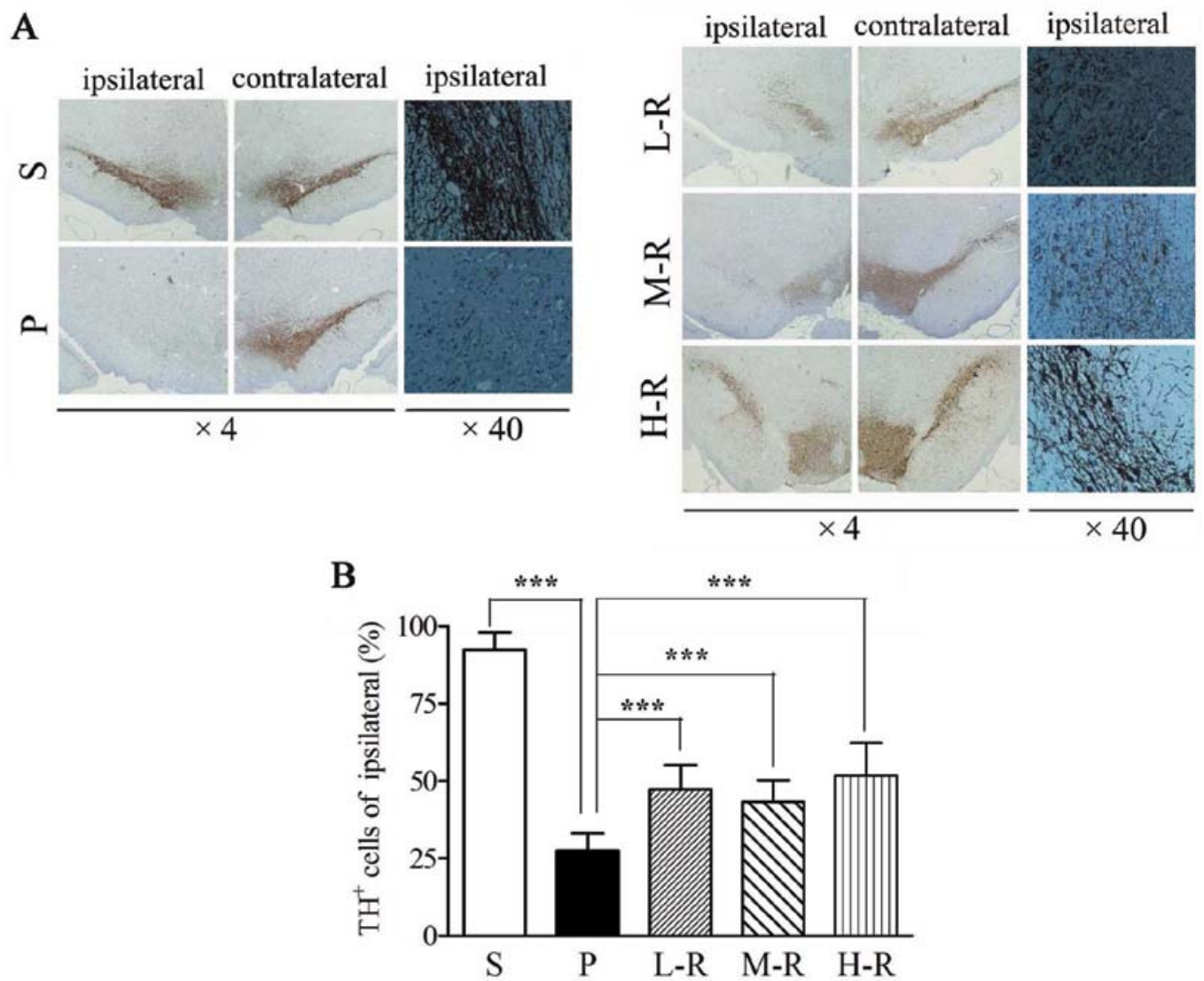

Figure 1. Rapamycin protects against the loss of dopaminergic neurons in a rat model of Parkinson's disease (PD). (A) Tyrosine hydroxylase (TH) immunohistochemistry of substantia nigra pars compacta (SNpc) sections from rats in the sham-operated (S), vehicle-pre-treated PD model (P), low-dose rapamycin $(\mathrm{L}-\mathrm{R})$, moderate-dose rapamycin $(\mathrm{M}-\mathrm{R})$, and high-dose rapamycin $(\mathrm{H}-\mathrm{R})$ pre-treated $\mathrm{PD}$ model groups. Images at $\mathrm{x} 4$ magnification show the ipsilateral and contralateral $\mathrm{TH}^{+}$neurons. Images at $\mathrm{x} 40$ magnification show the ipsilateral $\mathrm{TH}^{+}$neurons. (B) The number of ipsilateral $\mathrm{TH}^{+}$neurons was significantly lower in $\mathrm{PD}$ model rats $(\mathrm{P})$ compared to the sham-operated rats $(\mathrm{S})(\mathrm{p}<0.001)$. Pre-treatment with rapamycin significantly increased the number of ipsilateral $\mathrm{TH}^{+}$neurons as compared to pre-treatment with the vehicle $(\mathrm{P})($ all $\mathrm{p}<0.001)$. The number of ipsilateral $\mathrm{TH}^{+}$neurons is presented as a percentage of cells in the ipsilateral hemisphere vs. the contralateral one. Bars indicate the means $\pm \mathrm{SD}$ of the results. Each group consisted of 12 rats. ${ }^{* * *} \mathrm{p}<0.001$.
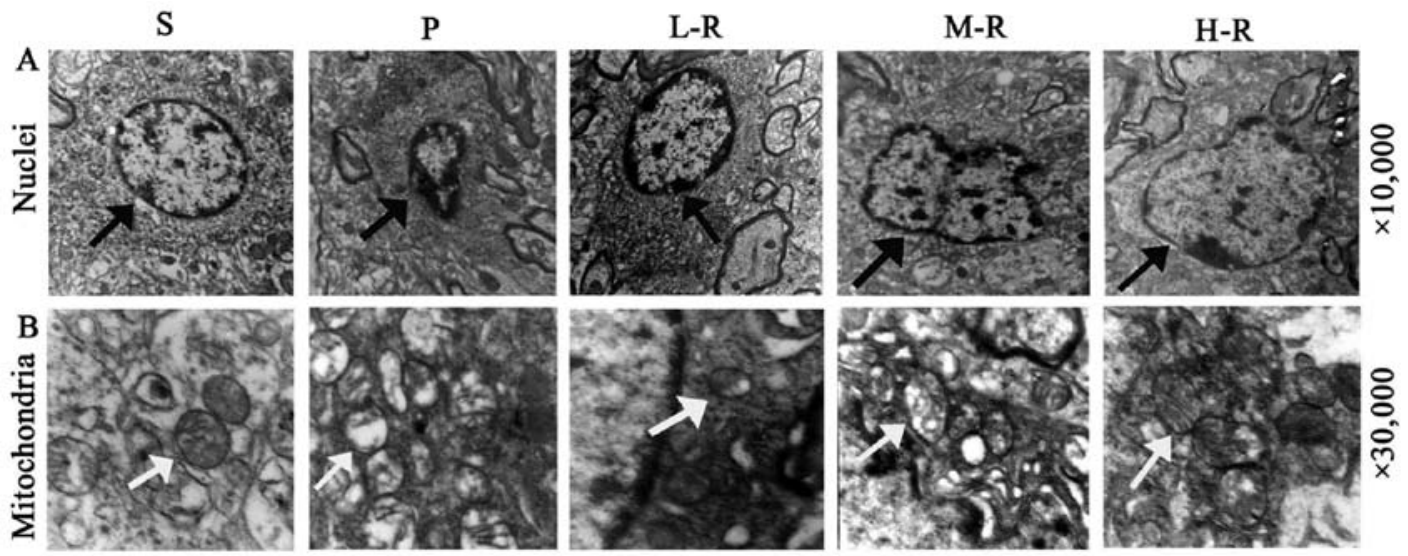

Figure 2. TEM ultrastructural micrographs of the rat striatum $(\mathrm{n}=6)$. Treatment with various doses [low-dose rapamycin $(\mathrm{L}-\mathrm{R})$, moderate-dose rapamycin $(\mathrm{M}-\mathrm{R})$ and high-dose rapamycin (H-R)] of rapamycin significantly improved the ultrastructures of (A) the nuclei (black arrows, x10,000 magnification) and (B) the mitochondria (white arrows, x30,000 magnification) as compared to pre-treatment with the vehicle (P). Sham-operated (S) rats showed intact nuclei and mitochondria.

those in the rats in group $\mathrm{S}(30.62 \pm 4.55$ vs. $13.87 \pm 3.32 \mathrm{nmol} / \mathrm{mg}$ protein, p<0.001) (Fig. 3A). However, the SOD $(6.76 \pm 1.93$ vs. $14.56 \pm 3.09 \mathrm{U} / \mathrm{mg}$ protein, $\mathrm{p}<0.001)$ and GSH-PX activity $(943.68 \pm 310.45$ vs. $1797.12 \pm 313.53 \mathrm{U} / \mathrm{mg}$ protein, $\mathrm{p}<0.001)$ was lower in group $\mathrm{P}$ compared to group S (Fig 3B and C).
Following pre-treatment with rapamycin, a reduction in MDA levels $(24.63 \pm 4.56 \mathrm{nmol} / \mathrm{mg}$ protein, $\mathrm{p}=0.003)$ and an increase in SOD $(10.86 \pm 3.12 \mathrm{U} / \mathrm{mg}$ protein, $\mathrm{p}=0.007)$ and $\mathrm{GSH}-\mathrm{PX}$ $(1324.65 \pm 251.63 \mathrm{U} / \mathrm{mg}$ protein, $\mathrm{p}=0.017)$ activity were observed in the rats in group L-R as compared with those in group $\mathrm{P}$ 

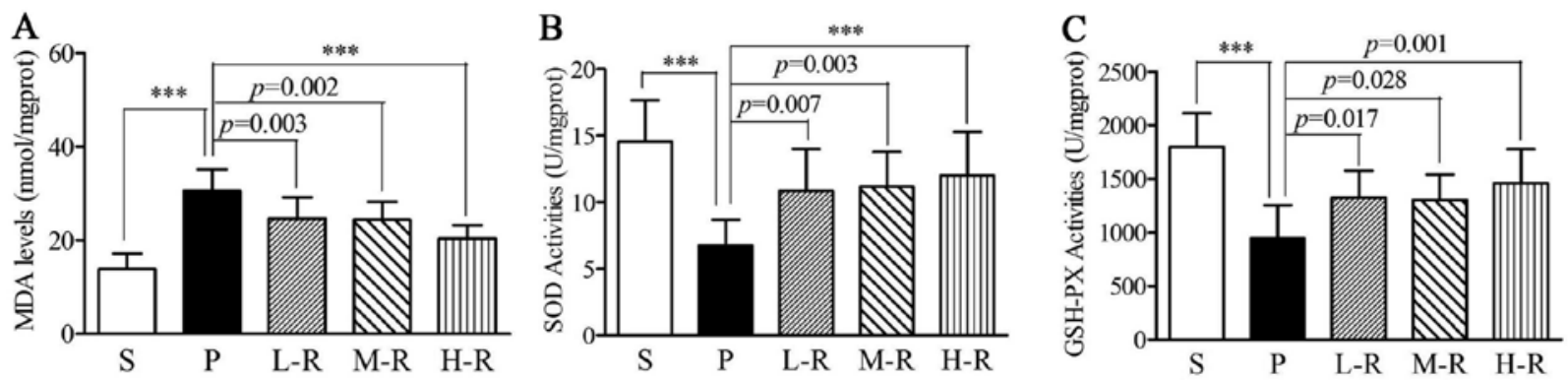

Figure 3. Levels of oxidative stress markers in rats with Parkinson's disease (PD). (A) Lower malondialdehyde (MDA) levels were observed in the groups pretreated with low ( $\mathrm{L}-\mathrm{R}, \mathrm{p}=0.003)$, moderate $(\mathrm{M}-\mathrm{R}, \mathrm{p}=0.002)$, and high $(\mathrm{H}-\mathrm{R}, \mathrm{p}<0.001)$ doses of rapamycin compared to the group pre-treated with the vehicle $(\mathrm{P})$. (B) Increased superoxide dismutase (SOD) activity was observed in the groups pre-treated with low (L-R, $\mathrm{p}=0.007)$, moderate (M-R, $p=0.003)$, and high (H-R, $\mathrm{p}<0.001)$ doses of rapamycin compared the group pre-treated with the vehicle $(\mathrm{P})$. (C) Increased glutathione peroxidase (GSH-PX) activity was observed in the groups pre-treated with low $(\mathrm{L}-\mathrm{R}, \mathrm{p}=0.017)$, moderate $(\mathrm{M}-\mathrm{R}, \mathrm{p}=0.028)$, and high $(\mathrm{H}-\mathrm{R}, \mathrm{p}=0.001)$ doses of rapamycin compared to the group pre-treated with the vehicle $(\mathrm{P})$. Bars indicate the means $\pm \mathrm{SD}$ of the results. Each group consisted of 12 rats. ${ }^{* * *} \mathrm{p}<0.001$.

(Fig. 3). In the rats in groups M-R and H-R, similar results were observed; the MDA levels (M-R, 24.40 $\pm 3.84 \mathrm{nmol} / \mathrm{mg}$ protein, $\mathrm{p}=0.002 ; \mathrm{H}-\mathrm{R}, 20.35 \pm 2.89 \mathrm{nmol} / \mathrm{mg}$ protein, $\mathrm{p}<0.001)$ were decreased and SOD $(\mathrm{M}-\mathrm{R}, 11.16 \pm 2.62 \mathrm{U} / \mathrm{mg}$ protein, $\mathrm{p}=0.003$; H-R, $12.01 \pm 3.25 \mathrm{U} / \mathrm{mg}$ protein, $\mathrm{p}<0.001)$ and GSH-PX (M-R, 1302.45 $\pm 236.38 \mathrm{U} / \mathrm{mg}$ protein, $\mathrm{p}=0.028$; H-R, $1456.90 \pm 320.03 \mathrm{U} / \mathrm{mg}$ protein, $\mathrm{p}=0.001$ ) activity was increased as compared to group P (Fig. 3). No statistically significant differences were observed in the levels of MDA and SOD and GSH-PX activity between the PD rats pre-treated with various doses of rapamycin ( $p>0.05$ in all cases).

Rapamycin alters the expression of Bcl-2 and Baxin a rat model of $P D$. In the rats in group P, RT-PCR analyses showed that the Bcl-2 mRNA levels $(33.40 \pm 5.20$ vs. $61.19 \pm 17.24, \mathrm{p}=0.001)$ were reduced and Bax mRNA levels $(57.86 \pm 12.97$ vs. $28.10 \pm 9.11$, $\mathrm{p}<0.001)$ were elevated as compared with those in the rats in group S (Fig. 4A-C). Pre-treatment with rapamycin significantly increased Bcl-2 (47.19 $\pm 9.97, \mathrm{p}=0.005)$ and decreased Bax $(44.65 \pm 7.78, \mathrm{p}=0.008)$ mRNA levels in group L-R as compared to pre-treatment with the vehicle (group P). The mRNA levels of Bcl-2 (M-R, 45.59 $\pm 8.53, \mathrm{p}=0.005$; H-R, $54.03 \pm 11.30, \mathrm{p}<0.001)$ and $\mathrm{Bax}(\mathrm{M}-\mathrm{R}, 46.70 \pm 6.98, \mathrm{p}=0.037$; $\mathrm{H}-\mathrm{R}, 41.85 \pm 8.49, \mathrm{p}=0.001)$ in groups $\mathrm{M}-\mathrm{R}$ and $\mathrm{H}-\mathrm{R}$ were also significantly different as compared with those in group $\mathrm{P}$ (Fig. 4A-C). Western blot analyses were performed to evaluate the protein expression of Bcl-2 and Bax in rapamycin-pretreated and vehicle-pre-treated PD rats. Further analyses revealed that pre-treatment with rapamycin significantly upregulated Bcl-2 and downregulated Bax protein expression compared to pre-treatment with the vehicle $(\mathrm{p}<0.05$ in all cases); these results were consistent with those from RT-PCR analyses (Fig. 4D-F). However, no statistically significant differences were observed in the mRNA and protein expression levels of Bcl-2 and Bax between the PD rats pre-treated with various doses of rapamycin ( $p>0.05$ in all cases).

Rapamycin reduces cytochrome c release and cleaved caspase-9 expression in a rat model of $P D$. Western blot analyses demonstrated that the release of cytochrome $c(42.86 \pm 7.73$ vs. $14.74 \pm 4.17, \mathrm{p}<0.001)$ and the expression of cleaved caspase- 9 $(42.72 \pm 8.77$ vs. $27.46 \pm 5.51, \mathrm{p}<0.001)$ were increased in the rats in group P, as compared with the rats in group S (Fig. 5). Pre-treatment with rapamycin significantly suppressed cytochrome $c$ release $(33.80 \pm 4.86, \mathrm{p}=0.004)$ and caspase -9 expression $(30.68 \pm 5.15, \mathrm{p}<0.001)$ in group $\mathrm{L}-\mathrm{R}$, as compared with the group pre-treated with the vehicle (group P) (Fig. 5). Pre-treatment with a moderate and high dose of rapamycin also reduced the release of cytochrome $c(\mathrm{M}-\mathrm{R}, 35.53 \pm 6.73$, $\mathrm{p}=0.029 ; \mathrm{H}-\mathrm{R}, 30.07 \pm 5.39, \mathrm{p}<0.001)$ and the expression of cleaved caspase-9 (M-R, 34.32 \pm 7.01 , $\mathrm{p}=0.026$; H-R, 32.28 \pm 6.42 , $\mathrm{p}=0.003$ ), as compared to the group pre-treated with the vehicle; no statistically significant differences were observed between groups L-R, M-R and H-R (p>0.05 in all cases) (Fig. 5).

\section{Discussion}

Oxidative stress and apoptosis play crucial roles in the pathogenesis of PD (4). Numerous efforts have been made to discover a therapeutic strategy to prevent the degeneration of dopaminergic neurons. The mTOR pathway regulates cell growth not only by regulating stress responses (21), but also by controlling mitochondrial energy metabolism (22). As a regulator of mTOR signaling, rapamycin protects against neuronal death by RTP801 blockage or through autophagy induction $(13,14,23)$. Consistently, in this study, a behavioral improvement and a reduced loss of $\mathrm{TH}^{+}$neurons were observed in the PD rats pre-treated with rapamycin. The aim of this study was to investigate the mitochondrial protective role of rapamycin in a rat model of $\mathrm{PD}$ by the analyses of oxidative stress and apoptosis in vivo.

Oxidative stress is a common characteristic in a number of current theories of the etiology of PD. Increases in oxidative stress precede the signs of neuronal degeneration (24), suggesting that oxidative stress may be an early component of neuronal loss. Smith and Cass $(4,25)$ indicated that oxidative stress is an early event in the course of dopamine depletion following 6-OHDA administration and glial cell line-derived neurotrophic factor (GDNF) reduced oxidative stress in a 6-OHDA model of PD. In addition, nucleolar disruption leads to oxidative damage through the mTOR pathway (19). To investigate whether rapamycin suppresses oxidative stress in a rat model of PD model, in this study, we detected the levels of MDA, which has been shown to be associated with mitochondrial peroxide activity (26). The 
A

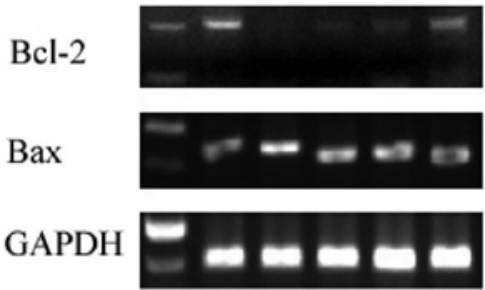

B

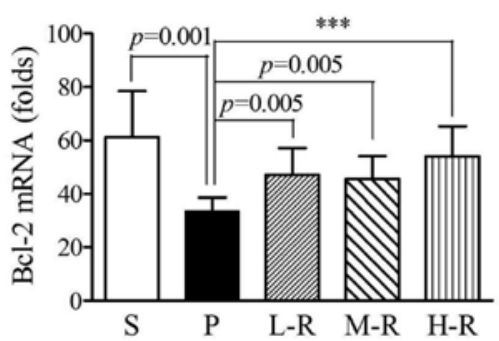

C

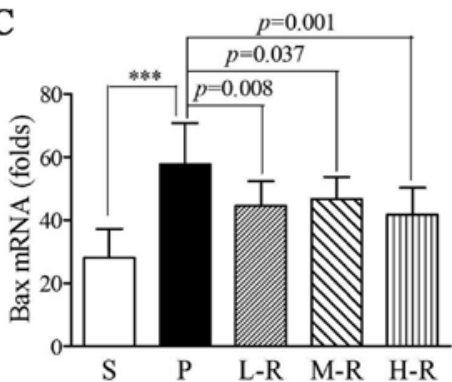

D

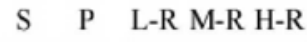

Bcl-2

Bax

GAPDH

E

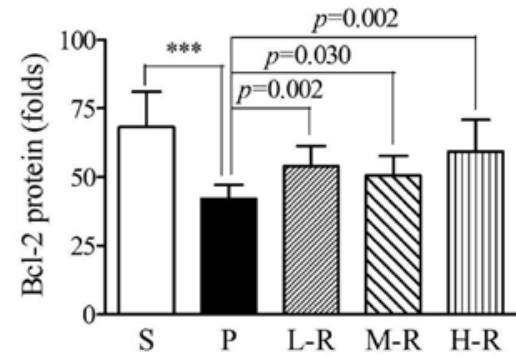

$\mathrm{F}$

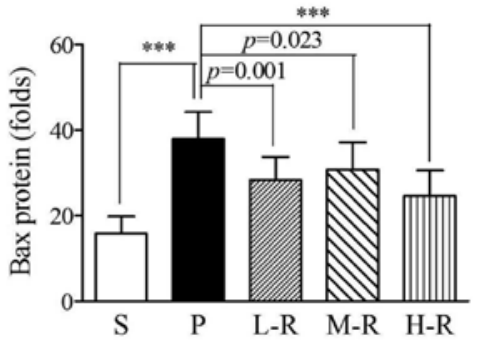

Figure 4. Expression of Bcl-2 and Bax in rats with Parkinson's disease (PD). (A-C) mRNA levels of Bcl-2 and Bax. (A) Bcl-2 and Bax RT-PCR gel electrophoresis patterns with GAPDH as the endogenous control. (B) Higher Bcl-2 (L-R, p=0.005; M-R, p=0.005; H-R, p<0.001) and (C) lower Bax (L-R, p=0.008; M-R, $\mathrm{p}=0.037 ; \mathrm{H}-\mathrm{R}, \mathrm{p}=0.001) \mathrm{mRNA}$ levels were observed in the groups pre-treated with various doses of rapamycin compared to the group pre-treated with the vehicle (P). (D-F) Protein expression of Bcl-2 and Bax. (D) Western blot analysis immunoreactive bands of GAPDH, Bcl-2 and Bax are shown. (E) Increased $\mathrm{Bcl}-2$ (L-R, p=0.002; M-R, p=0.030; H-R, p=0.002) and (F) reduced Bax ( $\mathrm{L}-\mathrm{R}, \mathrm{p}=0.001 ; \mathrm{M}-\mathrm{R}, \mathrm{p}=0.023 ; \mathrm{H}-\mathrm{R}, \mathrm{p}<0.001)$ protein expression was observed in the groups pre-treated with various doses of rapamycin compared to the group pre-treated with the vehicle $(\mathrm{P})$. Bars indicate the means $\pm \mathrm{SD}$ of the results. Each group consisted of 12 rats. ${ }^{* * *} \mathrm{p}<0.001$. L-R, low-dose rapamycin; M-R, moderate-dose rapamycin; H-R, high-dose rapamycin.

A

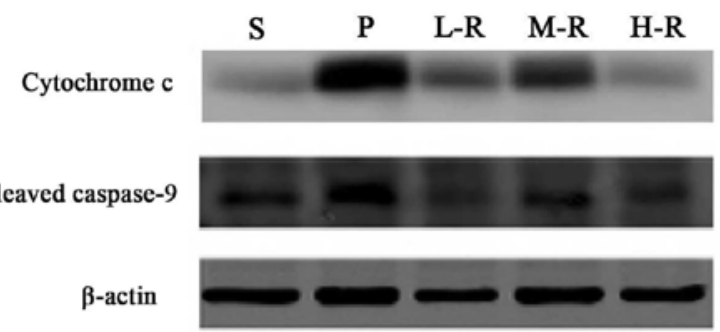

B

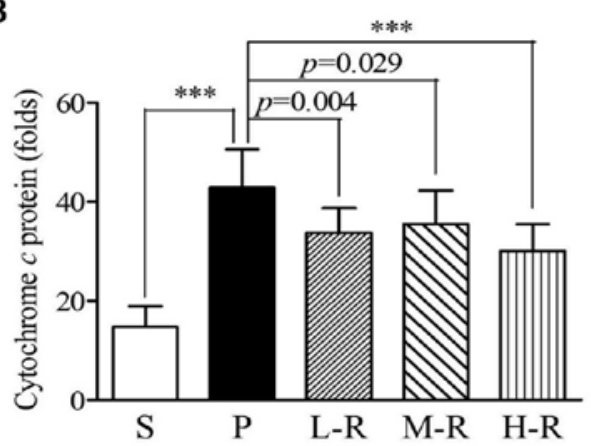

$\mathrm{C}$

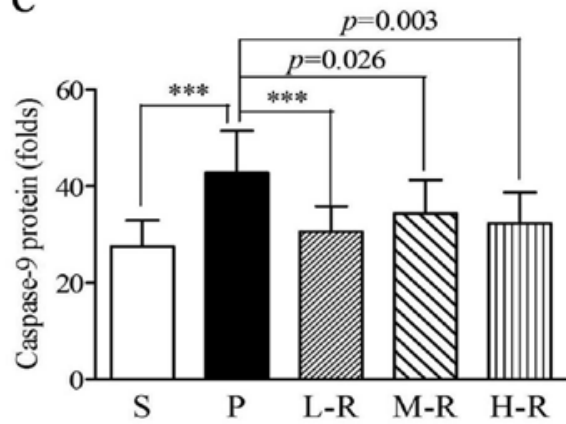

Figure 5. Cytochrome $c$ release and cleaved caspase-9 expression in rats with Parkinson's disease (PD). (A) Western blot analysis immunoreactive bands of $\beta$-actin, cytochrome $c$ and cleaved caspase-9 are shown. (B and C) Pre-treatment with various doses of rapamycin significantly reduced (B) cytochrome $c$ release (L-R, p=0.004; M-R, p=0.029; H-R, p<0.001) and (C) cleaved caspase-9 expression ( $\mathrm{L}-\mathrm{R}, \mathrm{p}<0.001 ; \mathrm{M}-\mathrm{R}, \mathrm{p}=0.026$; H-R, $\mathrm{p}=0.003)$ compared to pre-treatment with the vehicle $(\mathrm{P})$. Bars indicate the means $\pm \mathrm{SD}$ of the results. Each group consisted of 12 rats. ${ }^{* * *} \mathrm{p}<0.001$. 
levels of MDA were higher in the PD rats compared to the sham controls, but were significantly reduced following pre-treatment with rapamycin. However, in the rapamycin-pre-treated PD rats, the levels of antioxidant enzymes, such as SOD and GSH-PX were significantly increased. The changes in peroxide activity and antioxidant enzyme levels suggested that rapamycin reduced oxidative stress in vivo, which may protect against dopamine neuronal death and provide behavioral improvement in PD rats. Mitochondrial dysfunction is considered critical to the pathogenesis of PD, while oxidative stress and mitochondrial dysfunction reinforce each other and constitute a vicious circle (27). To assess the therapeutic effects on mitochondrial injuries, we performed TEM analyses and observed nuclei and mitochondria ultrastructural injuries in the PD rats. Pre-treatment with rapamycin led to a significant improvement in 6-OHDA-induced lesions, suggesting that rapamycin exerted a protective effect against mitochondrial dysfunction, possibly through the suppression of oxidative stress. Although the therapeutic effects in the high-dose group exhibited optimization, the results were not statistically different from those in the low- and moderate-dose groups. This was consistent with the results of behavioral analyses and the remaining $\mathrm{TH}^{+}$neuronal detections, suggesting that rapamycin protects against 6-OHDA-induced lesions, irrespective of the dose administered.

As is well known, apoptosis serves as a major cellular mechanism in the pathogenesis of PD. Previous studies have indicated that rapamycin protects against neuronal death or apoptosis in in vitro and in vivo models of PD (14). To evaluate the protective effects against apoptosis, the expression of proapoptotic or anti-apoptotic markers in rapamycin-pre-treated PD rats were detected by RT-PCR and western blot analysis. Mitochondrial-dependent apoptosis is finely regulated by a series of pro- and anti-apoptotic proteins, such as Bcl-2 family proteins that control the permeabilization of the mitochondrial outer membrane (28). Increased Bax and decreased Bcl-2 expression have been shown to reduce mitochondrial membrane potential and increase ROS production in neurons (29). The increased Bcl-2 expression and decreased Bax expression in rapamycin-pre-treated $\mathrm{PD}$ rats suggested that rapamycin may play a beneficial role by preventing neurons from subsequent pro-apoptotic insults. Cytochrome $c$ is usually found in the mitochondrial intermembrane space. The translocation of Bax to the mitochondria is followed by the permeabilization of the mitochondrial outer membrane, which results in the release of cytochrome $c$ and the activation of the apoptotic program. Its release leads to caspase- 9 cleavage and the initiation of apoptosis $(28,29)$. Thus, the reduced cytochrome $c$ release suggests that this protective effect of rapamycin may be predominantly mitochondrial-dependent and decreased cleaved caspase-9 may attenuate apoptotic responses to cellular oxidative stress. However, it cannot be excluded that rapamycin may protect against apoptosis initiated upstream of the mitochondria, such as Fas/Fas-ligand-mediated apoptosis.

Additionally, the protective effects of rapamycin against oxidative stress and apoptosis were not associated with the dose of administration. It is worth noting that mTOR forms 2 distinct physical and functional complexes, termed mTOR complex 1 (mTORC1) and mTOR complex 2 (mTORC2). mTORC1, which is sensitive to rapamycin, regulates translation and cell growth, whereas mTORC2 is insensitive to rapamycin (30). The
L-3,4-dihydroxyphenylalanine (L-DOPA)-mediated activation of mTORC1 has been shown to persist in mice that developed dyskinesia. Moreover, the mTORC1 inhibitor, rapamycin, prevented the development of dyskinesia without affecting the therapeutic efficacy of L-DOPA (31). In this study, the dissociation between the therapeutic effects and the administered dose suggested that all doses of rapamycin may selectively inhibit mTORC1 and have no interactions with mTORC2. The specific inhibition of $\mathrm{mTORC1}$ with a wide range of doses may provide evidence supporting its clinical application for the treatment of PD.

This study also raised the issue as to the mechanism involved in the protective effects of rapamycin against oxidative stress and apoptosis. Previous studies have provided evidence linking mitochondrial dysfunction, oxidative stress and energy depletion to neurodegenerative diseases, such as PD $(27,32)$. Autophagy has been suggested to be neuroprotective by enhancing the clearance of harmful protein aggregates, and the dysfunction of autophagy may result in abnormal mitochondrial function and oxidative stress (33). In addition, pre-treatment with rapamycin has been shown to protect against apoptosis through the induction of autophagy $(13,15,23)$. Therefore, it can by hypothesized that rapamycin reduces oxidative stress and protects against apoptosis, possibly by enhancing autophagy, as shown in our study.

In conclusion, this study demonstrates that rapamycin provides behavioral improvement and reduces the loss of dopaminergic neurons in PD. The neuroprotective properties of rapamycin arise from its capacity to reduce oxidative stress and mitochondrial injuries, which may consequently contribute to its anti-apoptotic effects. Our findings provide evidence for the employment of rapamycin as a therapeutic agent for the prevention of neuronal degeneration in PD. The antioxidant and anti-apoptotic mechanisms of rapamycin in PD remain to be elucidated in future studies.

\section{Acknowledgements}

We thank Mr. Wen-Xuan Zhou, Mr. Zhong-Ji Zhou, Mr. Ci-Yi Guo, Mr. Yu-Hai Chai and Mrs. Mei-Hua Ding for their assistance.

\section{References}

1. Dauer W and Przedborski S: Parkinson's disease: mechanisms and models. Neuron 39: 889-909, 2003.

2. Marras $\mathrm{C}$ and Lang A: Invited article: changing concepts in Parkinson disease: moving beyond the decade of the brain. Neurology 70: 1996-2003, 2008.

3. Levy OA, Malagelada C and Greene LA: Cell death pathways in Parkinson's disease: proximal triggers, distal effectors, and final steps. Apoptosis 14: 478-500, 2009.

4. Smith MP and Cass WA: Oxidative stress and dopamine depletion in an intrastriatal 6-hydroxydopamine model of Parkinson's disease. Neuroscience 144: 1057-1066, 2007.

5. Exner N, Lutz AK, Haass C and Winklhofer KF: Mitochondrial dysfunction in Parkinson's disease: molecular mechanisms and pathophysiological consequences. EMBO J 31: 3038-3062, 2012.

6. Yamato M, Kudo W, Shiba T, Yamada KI, Watanabe T and Utsumi H: Determination of reactive oxygen species associated with the degeneration of dopaminergic neurons during dopamine metabolism. Free Radic Res 44: 249-257, 2010.

7. Lee JE, Park JH, Shin IC and Koh HC: Reactive oxygen species regulated mitochondria-mediated apoptosis in PC12 cells exposed to chlorpyrifos. Toxicol Appl Pharmacol 263: 148-162, 2012. 
8. Hori A, Yoshida M, Shibata T and Ling F: Reactive oxygen species regulate DNA copy number in isolated yeast mitochondria by triggering recombination-mediated replication. Nucleic Acids Res 37: 749-761, 2009

9. Noda T and Ohsumi Y: Tor, a phosphatidylinositol kinase homologue, controls autophagy in yeast. J Biol Chem 273: 3963-3966, 1998.

10. Sudarsanam S and Johnson DE: Functional consequences of mTOR inhibition. Curr Opin Drug Discov Devel 13: 31-40, 2010.

11. Ravikumar B, Duden R and Rubinsztein DC: Aggregate-prone proteins with polyglutamine and polyalanine expansions are degraded by autophagy. Hum Mol Genet 11: 1107-1117, 2002.

12. Ravikumar B, Vacher C, Berger Z, Davies JE, Luo S, Oroz LG, et al: Inhibition of mTOR induces autophagy and reduces toxicity of polyglutamine expansions in fly and mouse models of Huntington disease. Nat Genet 36: 585-595, 2004.

13. Pan T, Kondo S, Zhu W, Xie W, Jankovic J and Le W: Neuroprotection of rapamycin in lactacystin-induced neurodegeneration via autophagy enhancement. Neurobiol Dis 32: 16-25, 2008.

14. Malagelada C, Jin ZH, Jackson-Lewis V, Przedborski S and Greene LA: Rapamycin protects against neuron death in in vitro and in vivo models of Parkinson's disease. J Neurosci 30: $1166-1175,2010$

15. Ravikumar B, BergerZ, VacherC,O'Kane CJ and Rubinsztein DC: Rapamycin pre-treatment protects against apoptosis. Hum Mol Genet 15: 1209-1216, 2006.

16. Marobbio CM, Pisano I, Porcelli V, Lasorsa FM and Palmieri L: Rapamycin reduces oxidative stress in frataxin-deficient yeast cells. Mitochondrion 12: 156-161, 2012.

17. Siddiqui A, Hanson I and Andersen JK: Mao-B elevation decreases parkin's ability to efficiently clear damaged mitochondria: protective effects of rapamycin. Free Radic Res 46: 1011-1018, 2012

18. Kofman AE, McGraw MR and Payne CJ: Rapamycin increases oxidative stress response gene expression in adult stem cells. Aging (Albany NY) 4: 279-289, 2012.

19. Rieker C, Engblom D, Kreiner G, Domanskyi A, Schober A, Stotz S, et al: Nucleolar disruption in dopaminergic neurons leads to oxidative damage and parkinsonism through repression of mammalian target of rapamycin signaling. J Neurosci 31: 453-460, 2011
20. Paxinos G and Watson C: The Rat Brain in Stereotaxic Coordinates. 6th edition. Academic Press, London, 2007.

21. Schieke SM, Phillips D, McCoy JP Jr, Aponte AM, Shen RF, Balaban RS, et al: The mammalian target of rapamycin (mTOR) pathway regulates mitochondrial oxygen consumption and oxidative capacity. J Biol Chem 281: 27643-27652, 2006.

22. Wullschleger S, Loewith R and Hall MN: TOR signaling in growth and metabolism. Cell 124: 471-484, 2006.

23. Pan T, Rawal P, Wu Y, Xie W, Jankovic J and Le W: Rapamycin protects against rotenone-induced apoptosis through autophagy induction. Neuroscience 164: 541-551, 2009.

24. Hall ED, Detloff MR, Johnson K and Kupina NC: Peroxynitritemediated protein nitration and lipid peroxidation in a mouse model of traumatic brain injury. J Neurotrauma 21: 9-20, 2004.

25. Smith MP and Cass WA: GDNF reduces oxidative stress in a 6-hydroxydopamine model of Parkinson's disease. Neurosci Lett 412: 259-263, 2007.

26. Long J, Liu C, Sun L, Gao H and Liu J: Neuronal mitochondrial toxicity of malondialdehyde: inhibitory effects on respiratory function and enzyme activities in rat brain mitochondria. Neurochem Res 34: 786-794, 2009.

27. Onyango IG: Mitochondrial dysfunction and oxidative stress in Parkinson's disease. Neurochem Res 33: 589-597, 2008.

28. Martinou JC and Youle RJ: Mitochondria in apoptosis: Bcl-2 family members and mitochondrial dynamics. Dev Cell 21 92-101, 2011.

29. Kirkland RA and Franklin JL: Bax, reactive oxygen, and cytochrome c release in neuronal apoptosis. Antioxid Redox Signal 5: 589-596, 2003

30. Bhagwat SV and Crew AP: Novel inhibitors of mTORC1 and mTORC2. Curr Opin Investig Drugs 11: 638-645, 2010

31. Santini E, Heiman M, Greengard P, Valjent E and Fisone G: Inhibition of mTOR signaling in Parkinson's disease prevents L-DOPA-induced dyskinesia. Sci Signal 2: ra36, 2009.

32. Lin MT and Beal MF: Mitochondrial dysfunction and oxidative stress in neurodegenerative diseases. Nature 443: 787-795, 2006.

33. Lee J, Giordano S and Zhang J: Autophagy, mitochondria and oxidative stress: cross-talk and redox signalling. Biochem J 441: 523-540, 2012 